\title{
Conformation of Single Poly(methyl methacrylate) Chains in an Ultra-Thin Film Studied by Scanning Near-Field Optical Microscopy
}

\author{
By Hiroyuki AOKI, ${ }^{*}$ Sayuri MoRITA, Ryojun SEKINE, and Shinzaburo ITO
}

The conformation of a single poly(methyl methacrylate) (PMMA) chain was studied by scanning near-field optical microscopy (SNOM). Here we discuss the conformation of PMMA in the confined geometry of the thin film with a thickness less than the unperturbed chain dimension in the bulk state. The direct observation by SNOM was performed for individual fluorescence-labeled PMMA chains dispersed in the thin films of the unlabeled PMMA prepared by the spin coating and Langmuir-Blodgett techniques. We examined the effect of the constraint in the height direction on the radius of gyration in the direction parallel to the film surface, $R_{\mathrm{xy}}$, i.e., the chain dimension normal to the confinement. In the thickness range of $1-100 \mathrm{~nm}, R_{\mathrm{xy}}$ in an ultra-thin film was not significantly altered from that in the bulk state, indicating that the PMMA chain has lowered interchain entanglement in the ultra-thin film.

KEY WORDS: Single Polymer Chain / Thin Film / Chain Conformation / Scanning Near-Field Optical Microscopy / Fluorescence

Microscopy /

Polymer thin films have been widely used in various fields (e.g., coatings and adhesives) and have been extensively explored from both the fundamental and applied points of view. The physical properties of the polymeric thin film differ from those in the three-dimensional bulk state when the thickness is less than the unperturbed dimension of the polymer chain. ${ }^{1-8}$ The effect of the interaction between the polymer chain and the interface is a crucial factor to determine the characteristic properties of the thin film. As well as the interface effect, the effect of the reduced degree of freedom of a polymer chain due to the spatial confinement is also important. The properties of the individual chain and the interaction among the surrounding chains would be altered from the bulk state. The conformation of the single polymer chain is one of the most fundamental issues to understand the physics of the polymer thin film; therefore, it has attracted much attention and has been extensively studied by many researchers. The scaling theory predicted that the radius of gyration, $R_{\mathrm{g}}$, of the polymer chain with the degree of polymerization of $N$ would be expressed as $R_{\mathrm{g}} \sim N^{0.5}$ for the two-dimensional chain, indicating that the two-dimensional chain would be segregated. ${ }^{9}$ Computer simulation studies have shown that for the polymer chain restricted between two walls separated by less than the bulk $R_{\mathrm{g}}$ the chain dimension parallel to the surface is not dependent on the gap between the walls and that the value of $R_{\mathrm{g}}$ is scaled by a factor of $\sim 0.5$ in the two-dimensional limit. ${ }^{10-15}$ Although the experimental method to study the conformation of the polymer chain in the ultra-thin film is limited because of the weak signal from the low sample volume, the small angle neutron scattering (SANS) is a powerful technique to probe the chain conformation. Kraus et al. studied the chain morphology of polystyrene (PS) in the thin film of the regular and deuterated PS by SANS experiments. ${ }^{16}$ The radius of gyration, $R_{\mathrm{g}}$, in the parallel direction to the film surface increased with the decrease of the film thickness for the thickness range of less than $6 R_{\mathrm{g}}$, and a similar thickness dependence of the chain conformation in the ultra-thin film has been reported by other groups. ${ }^{17,18}$ In contrast, Jones et al. reported the different behavior of the chain dimension of PS in the spin-cast thin film. They showed that the PS chain in an ultra-thin film takes a Gaussian conformation in the lateral dimension and the in-plane $R_{\mathrm{g}}$ is similar to that in the unperturbed state. ${ }^{19,20}$ In contrast to the scattering techniques used in previous studies, the real-space imaging of the individual polymer chains provides us the direct information on the chain conformation in the ultra-thin film. Several studies have been performed using polymer monolayer systems as models of the polymer chain in two dimensions. Maier and Radler studied the DNA chains adsorbed to a cationic lipid membrane. $^{21,22}$ In the highly concentrated condition, which can be regarded as the two-dimensional system, they found that the single DNA molecule took a collapsed conformation. Ito and his co-workers revealed the contracted conformation of poly(isobutyl methacrylate) in the monolayer from the energy transfer spectroscopy and the direct observation of the conformation of the single chain in a Langmiur-Blodgett film. ${ }^{23,24}$ On the other hand, some research groups recently reported the expanded conformation contradictorily to the above segregated model of the two-dimensional macromolecule. $^{25,26}$ Thus, a clear picture of the chain conformation in the restricted geometry has not been obtained.

The current study focuses on the effect of the spatial confinement on the chain conformation. The conformation of the single polymer chain is directly observed for the thin film 
with a thickness less than the unperturbed dimension. Recently, atomic force microscopy (AFM) has been used to study single polymer chain. ${ }^{27-29}$ The nanometric spatial resolution of AFM allows clear imaging of the single chain contour on an atomically flat substrate. However, AFM observes the isolated chain from the others at an extremely dilute condition and is applicable to measurement of the most surface of the specimen; therefore, the contour of an individual macromolecule embedded in the bulk medium cannot be tracked. Fluorescence microscopy is one of the most versatile techniques to obtain the information from the single polymer chain. The fluorescence labeling is the key feature for observation of the single chain. Since the fluorescence moieties are selectively introduced to a single chain in the bulk system, the conformation of the single fluorescent chain can be distinguished from the surrounding unlabeled ones in the fluorescence microscopy image. Therefore, the fluorescence microscopy is a powerful technique to observe the single polymer chain in the bulk system. However, the optical microscopy is not applicable to analysis of a structure less than $\sim 250 \mathrm{~nm}$ because the spatial resolution is limited by the diffraction barrier, and it has been used to observe huge biological macromolecules such as DNA. ${ }^{21,22}$ Scanning near-field optical microscopy (SNOM) has been developed to provide the optical information with the high spatial resolution beyond the diffraction limit of light. ${ }^{30-36}$ SNOM is a scanned probe microscopy, which uses the probe tip having an aperture smaller than the wavelength of light. The optical near-field generated at the probe end is confined in the vicinity of the aperture, which enables us to focus the light in a nanometric area. Therefore, the fluorescence imaging by SNOM is a promising technique for the observation of the contour of a single polymer chain in a bulk state. ${ }^{24,37}$ Here we present the direct measurement of the real-space image of the poly(methyl methacrylate) (PMMA) chain in thin films by SNOM. The chain conformation is discussed for the PMMA chain with a reduced degree of freedom in the height direction by a confinement in the ultra-thin film thinner than the unperturbed chain dimension. The thickness dependence is examined in a wide thickness range of the sample film from the monolayer $(\sim 1 \mathrm{~nm})$ to a thickness comparable to the unperturbed dimension.

\section{EXPERIMENTS}

Perylene-labeled PMMA (PMMA-Pe) was synthesized by radical copolymerization of methyl methacrylate and 3perylenylmethyl methacrylate, ${ }^{38}$ the chemical structure of which is shown in Figure 1. Fractional precipitation of the obtained polymer from toluene/methanol was carried out to obtain the PMMA-Pe with a relatively narrow molecular weight distribution. The molecular weight was characterized by size exclusion chromatography (SEC) with the exclusion limit of $2 \times 10^{7}$ (Shodex), which was calibrated by PMMA standards (Scientific Polymer Science). The weight- and numberaverage molecular weights $\left(M_{\mathrm{w}}\right.$ and $\left.M_{\mathrm{n}}\right)$ were evaluated to be $5.14 \times 10^{6}$ and $4.16 \times 10^{6}$, respectively, and the polydispersity

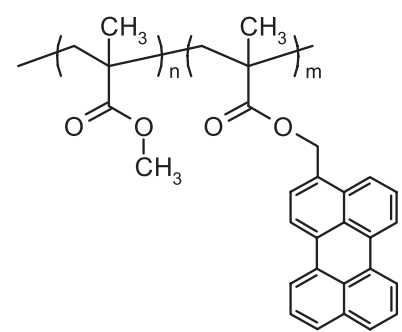

Figure 1. Chemical structure of perylene-labeled PMMA. The molar fraction of the dye-labeled unit, $\mathrm{m} /(\mathrm{m}+\mathrm{n})$, is evaluated to be $7.7 \times 10^{-3}$ from the UV-vis absorption.

index, $M_{\mathrm{w}} / M_{\mathrm{n}}$, was 1.23 . The molar fraction of the perylene moiety was $0.77 \%$, indicating that about 300 perylene molecules were tagged to each PMMA-Pe chain. A small amount of PMMA-Pe was added to a toluene solution of the unlabeled PMMA, and the resulting mixture was spin-cast on a quartz cover slip to form a thin film. The concentration of the polymer solution and the spinning rate were adjusted to obtain the films with a thickness of 7-100 nm. The sample films were annealed at $150^{\circ} \mathrm{C}$ for several days to reach the equilibrium before the SNOM measurement. A thickness of less than $7 \mathrm{~nm}$ was not available by the spin-coating method because of dewetting of PMMA from the substrate during the annealing. Therefore, the Langmuir-Blodgett technique was employed to prepare the monolayer of PMMA with the thickness of $c a$. $1 \mathrm{~nm} .{ }^{39}$ A benzene solution of the labeled/unlabeled PMMA mixture was spread dropwise on the surface of purified water (NANOpure II, Barnstead) at $20^{\circ} \mathrm{C}$. After the evaporation of the solvent the monolayer was compressed by a Teflon bar up to the surface pressure of $5 \mathrm{mN} \mathrm{m}^{-1}$ at a speed of $10 \mathrm{~mm} \mathrm{~min}^{-1}$. The monolayer of the PMMA was transferred by the horizontal dipping onto the glass substrate. For the evaluation of the spatial resolution of the apparatus, the single rhodmaine $B$ molecule was used to examine the point-spread function. Rhodamine B was spin-coated on a clean cover slip from the ethanol solution at the concentration of $10^{-9} \mathrm{M}$.

In the measurement by SNOM, we used a commercially available instrument ( $\alpha$-SNOM, WITec) equipped with a cantilever probe with an aperture at the end of the pyramidal tip, the diameter of which was $60 \mathrm{~nm}$. A He-Cd laser (442 nm, IK5351R-D, Kimmon Electric) and a diode-pumped solid state laser (532 nm, GSHG-3010, Kochi Toyonaka Giken) were used as the light sources for the excitation of perylene and rhodamine $\mathrm{B}$, respectively. The excitation laser beam was delivered to the SNOM head through a single mode optical fiber and focused on the backside of the aperture to generate the optical near-field. Besides the excitation light for the fluorescence measurement, the laser beam at $780 \mathrm{~nm}$ was incident on the cantilever. The reflected beam was detected with a four-segmented photo-diode to measure the cantilever deflection, from which the force between the sample and the SNOM probe was evaluated. The SNOM probe was scanned in contact with the sample surface at a constant force by regulating the vertical position of the probe tip, resulting in the surface topography image. While scanning with the SNOM 
probe, the fluorescence from perylene was collected with a microscope objective $(60 \times, 0.8 \mathrm{NA}$, Nikon), passed through a long-pass filter (AELP454, Omega Optical), and detected with a photomultiplier (H8631, Hamamatsu Photonics).

\section{RESULTS AND DISCUSSION}

For the quantitative discussion of the conformation of the chain contour, the spatial resolution is a parameter of utmost importance. First, the resolution of the SNOM system was evaluated from the measurement of a single dye molecule, which can be considered as an infinitely small object compared to the size of the SNOM probe. Therefore, the observed pattern for a single molecule corresponds to the point spread function of the imaging system. The spatial resolution of the probe is defined as the full width at half maximum (fwhm) for the individual molecules. Figure 2 indicates the fluorescence images of single rhodamine B molecules observed by the conventional confocal microscopy and SNOM. Each molecule was observed as a bright spot on the micrograph, and it showed discrete blinking and photo-bleaching during the measurement. This indicates that the observed bright spots correspond to the individual dye molecules. The single rhodamine molecule in the confocal image was observed as a circular spot with a diameter of $300 \mathrm{~nm}$, which shows the diffraction-limited spatial resolution of about the half of the wavelength of light. SNOM enables us to observe the individual molecules as small as $75 \mathrm{~nm}$, indicating that the spatial resolution is sufficiently high to observe the conformation of the single polymer chain with a relatively high molecular weight. Since the resolution is greatly dependent on the probe tip, all of the samples were observed using the identical probe used to obtain the SNOM image shown in Figure 2b.

Figure 3 shows the fluorescence SNOM image of the PMMA thin films with a thickness of $15 \mathrm{~nm}$, where the perylene-labeled PMMA chains were dispersed in an unlabeled polymer matrix at different concentrations. The topographic image obtained simultaneously showed a smooth and featureless surface with a root-mean-squared roughness less than $0.3 \mathrm{~nm}$ (the image is not shown here), indicating a homogeneous thin film without any defect. The thickness of the sample film was evaluated from the height difference between the sample surface and the substrate exposed by scratching the film. Since the optical near-field generated from the SNOM (a)

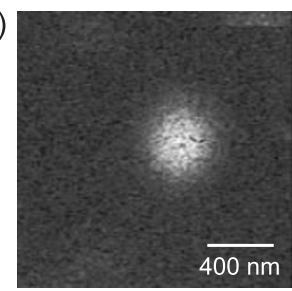

(b)

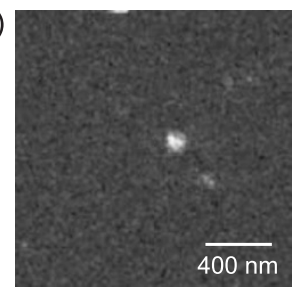

(c)
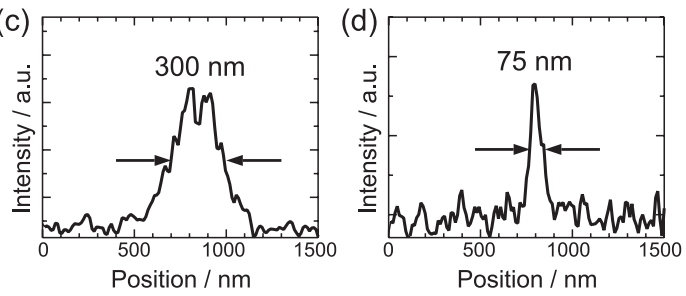

Figure 2. Fluorescence SNOM images for the single rhodamine B molecule observed by confocal microscopy (a) and SNOM (b). The fluorescence intensity profiles are shown in the lower panels $c$ and $d$ for the confocal and SNOM images, respectively. The rhodamine $B$ molecule in panel b was observed as circular spots with the diameter of $75 \mathrm{~nm}$, indicating the spatial resolution of $\lambda / 7$, where $\lambda$ is the excitation wavelength

probe penetrates into the sample film, the labeled PMMA chain embedded in the unlabeled PMMA matrix can be selectively imaged. At a high concentration of the labeled PMMA, the fluorescence signal of the labeled chain was observed uniformly from the whole area. At an extremely low concentration, the labeled polymer was observed as discrete bright spots as shown in Figure 3, and the number of fluorescence spots in the SNOM image decreased with the decrease of the concentration of the labeled polymer.

We verified that each fluorescence spot corresponds to the individual PMMA-Pe chain. Figure 4a shows the relationship between the dye-labeled chain fraction in the PMMA film and the number of fluorescence spots in a unit area in the low concentration region of the labeled PMMA. The number of perylene-labeled PMMA chains can be estimated from the concentration of the labeled PMMA in the unlabeled PMMA matrix. The solid line indicates the number of single perylenelabeled PMMA chains in a unit area of $1 \times 1 \mu \mathrm{m}^{2}$ calculated from the concentration of the labeled polymer. In a low concentration range, the number of observed fluorescence spots was in good agreement with the calculated value for the PMMA-Pe chain. This indicates that each fluorescence spot
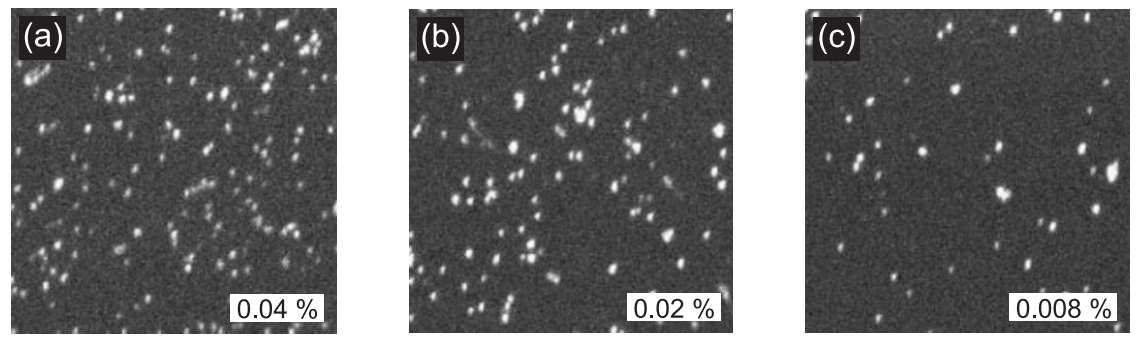

Figure 3. Fluorescence SNOM images of the PMMA-Pe chains dispersed in the unlabeled PMMA matrix. The concentration of the labeled chain is 0.04 (a), $0.02(\mathrm{~b})$, and $0.008 \%$ (c). The observed area was $20 \times 20 \mu \mathrm{m}^{2}$. 
(a)

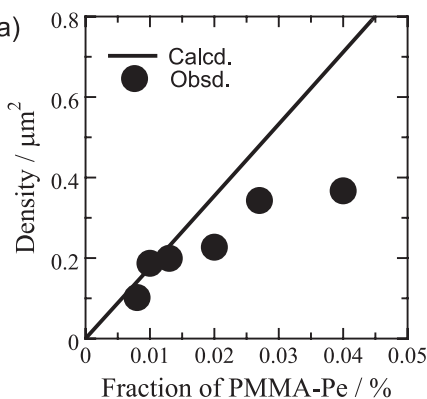

(b)

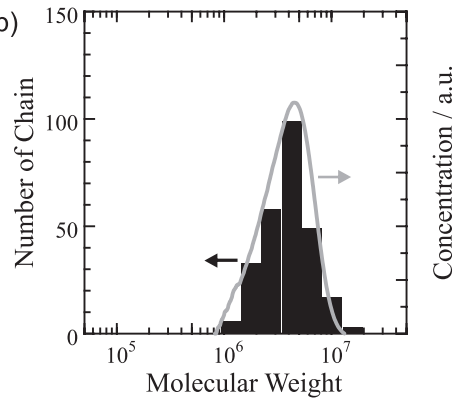

Figure 4. Number density of the observed fluorescence spot and the calculated value of the PMMA-Pe chain (a) and the histogram of the integrated fluorescence intensity for each PMMA-Pe chain (b). In panel a, the closed circles and the solid line indicate the observed and calculated values, respectively. In panel b, the thick bars are the intensity histogram, and the gray solid curve indicates the molecular weight distribution observed by SEC, where the chain number concentration was plotted against the molecular weight.

corresponds to the individual PMMA-Pe chain. For the sample with a thickness of $15 \mathrm{~nm}$, at a concentration higher than $0.015 \%$ the density of the fluorescence spot was less than the calculated line as shown in Figure 4a; therefore, for the 15-nm thick film, the observation of the single chain was performed under a concentration condition of $0.015 \%$. This threshold concentration is dependent on the film thickness. Even at the same concentration of PMMA-Pe in the unlabeled matrix, the number of labeled chains in an observed unit area is proportional to the sample thickness. Hence, the concentration dependence of the area density of the fluorescence spot was carefully examined for each film thickness, and the SNOM measurement was performed at a sufficiently low concentration of the labeled chain to ensure the single chain observation.
Moreover, the integrated fluorescence intensity from each spot was examined. The number of perylene molecules in the single PMMA-Pe chain is proportional to the molecular weight, because the perylene moiety was randomly introduced to the polymer chain. The low fraction of the dye moiety in each chain results in the negligible interaction among the perylene molecules. Thus the fluorescence intensity from the single chain is proportional to its molecular weight:

$$
I_{i}=k M_{i}
$$

where $I_{i}$ and $M_{i}$ are the integrated fluorescence intensity and the molecular weight for the $i$-th chain, respectively, and $k$ is a constant determined by the fluorescence quantum yield of perylene, the detection efficiency of the signal collection, etc. Therefore, assuming that the bright spot corresponds to the single chain, the histogram of the fluorescence intensity corresponds to the molecular weight distribution. Figure $4 \mathrm{~b}$ indicates the fluorescence intensity histogram and the molecular weight distribution observed by SEC. The emission intensity histogram and the molecular weight distribution are in good agreement. This data also supports the single PMMAPe chain observation in the SNOM image. It should be noted that this good agreement between the fluorescence intensity histogram and the molecular weight distribution indicates that the molecular weight of the single chain can be evaluated by the SNOM measurement.

Figure 5 shows the high-resolution SNOM images of the single PMMA-Pe chains in the thin film. The molecular weight of each chain was estimated to be $c a .4 .0 \times 10^{6}$ from the comparison between the fluorescence intensity and the molecular weight distribution curve shown in Figure 4b. As clearly shown in Figure 5 the polymer chains with similar molecular weight showed various shapes, and a part of the chains took a stretched conformation as shown in Figure $5 \mathrm{~d}$ This indicates the broad conformational distribution of the flexible PMMA chain. The chain dimension in the $x-y$ plane weighted by the fluorescence intensity, $R_{\mathrm{xy}}$, was evaluated for each PMMA-Pe chain in the SNOM image according to the following equation.

$$
R_{\mathrm{xy}}^{2}=\frac{\sum_{j} I_{j}\left(\mathbf{r}_{j}-\mathbf{r}_{\mathrm{c}}\right)^{2}}{\sum_{j} I_{j}},
$$
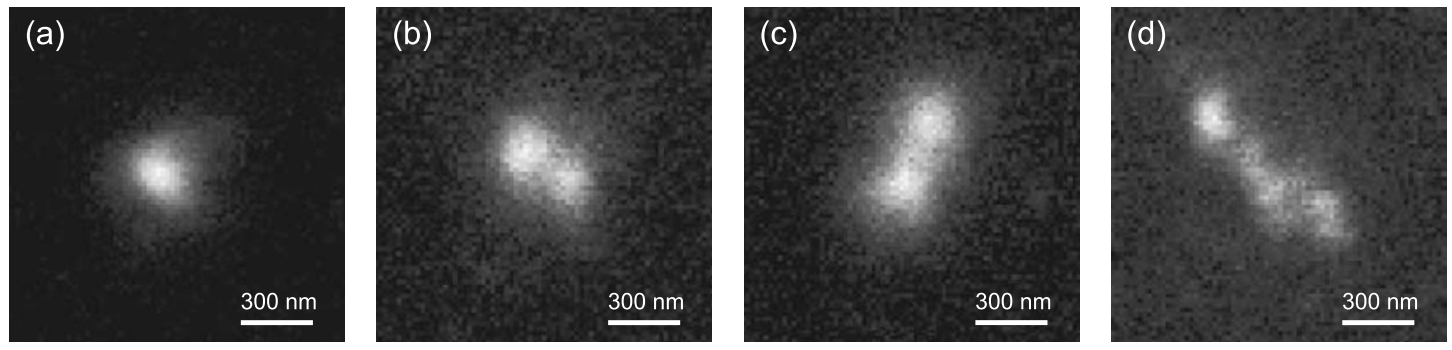

Figure 5. SNOM images of the single PMMA-Pe chains in an ultra-thin film. The scanned area is $1.4 \times 1.4 \mu \mathrm{m}^{2}$ for each image. The molecular weight $(M)$ and the value of $R_{\mathrm{xy}}$ for each chain were evaluated as follows: (a) $M=4.2 \times 10^{6}$ and $R_{\mathrm{xy}}=122 \mathrm{~nm}$; (b) $M=4.2 \times 10^{6}$ and $R_{\mathrm{xy}}=160 \mathrm{~nm}$; (c) $M=4.0 \times 10^{6}$ and $R_{\mathrm{xy}}=180 \mathrm{~nm} ;$ (d) $M=3.6 \times 10^{6}$ and $R_{\mathrm{xy}}=256 \mathrm{~nm}$. 


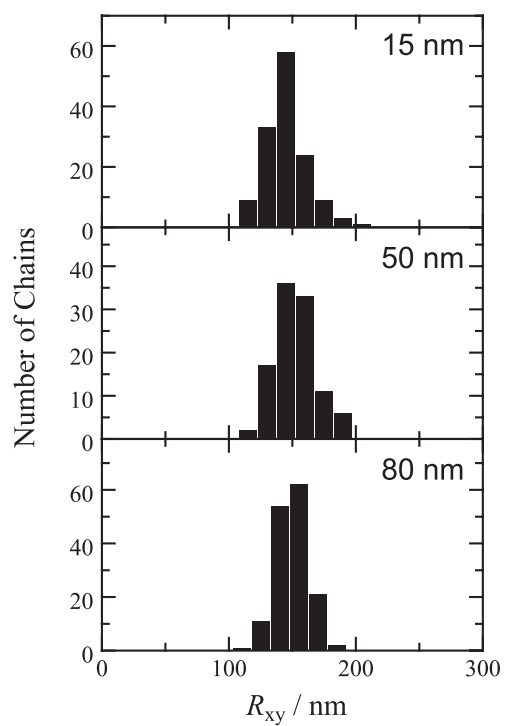

Figure 6. Histogram of the lateral chain dimension for the PMMA-Pe chains in the ultra-thin films with the thickness of 15,50 , and $80 \mathrm{~nm}$. The PMMA chains with a molecular weight of $4 \times 10^{6}$ were selected in the SNOM images and analyzed to construct the histogram.

where $\mathbf{r}_{j}$ and $I_{j}$ are the position vector and the fluorescence intensity for the $j$-th pixel in the SNOM image, and $\mathbf{r}_{c}$ is the position vector of the center of mass defined as

$$
\mathbf{r}_{\mathrm{c}}=\sum_{j} I_{j} \mathbf{r}_{j}
$$

Since the dye moiety was randomly introduced in the PMMAPe chain as mentioned above, the fluorescence intensity at each pixel is proportional to the chain segment number therein. Therefore, $R_{\mathrm{xy}}$ is corresponding to the radius of gyration of the PMMA-Pe chain projected in the x-y plane parallel to the film surface. For example, $R_{\mathrm{xy}}$ for the PMMA chains shown in Figure $5 \mathrm{a}-\mathrm{d}$ is estimated to be 122, 160, 180, and $256 \mathrm{~nm}$, respectively.

Here we discuss the thickness dependence of the chain dimension in the film plane. At first, we have to consider the relationship between the unperturbed dimension of the PMMA chain and the thickness range of the film used in the current work. By a SANS study, O'Reilly et al. showed that the radius of gyration of PMMA in the bulk state can be expressed by an empirical equation: $R_{\mathrm{g}}=0.025 M_{\mathrm{w}}{ }^{1 / 2} / \mathrm{nm} .{ }^{40}$ The PMMA sample used here is estimated to have an $R_{\mathrm{g}}$ of $56 \mathrm{~nm}$ considering the molecular weight. The maximum thickness of the spin-cast film used here is $100 \mathrm{~nm}$, which is the comparable to the unperturbed chain dimension in the bulk state. Figure 6 shows the histogram of $R_{\mathrm{xy}}$ for the PMMA-Pe chain in the films with a thickness of 15,50 , and $80 \mathrm{~nm}$. This corresponds to the probability distribution function of the chain dimension projected to the $\mathrm{x}-\mathrm{y}$ plane, which cannot be obtained from scattering experiments. The histogram for each thickness has a peak value at $R_{\text {xy }}$ of $140 \mathrm{~nm}$ and the width of the distribution of about $30 \mathrm{~nm}$. Figure 7 summarizes the thickness dependence of the average value of $R_{\mathrm{xy}}$. The filled circles in Figure 7 indicate

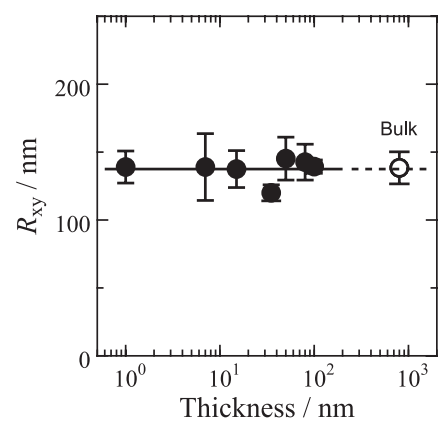

Figure 7. Thickness dependence of the chain dimension of PMMA in the parallel direction to the film surface. The filled circle indicates the value of $R_{\mathrm{xy}}$ for the ultra-thin film. The error bar indicates the standard deviation evaluated from the histogram of $R_{x y}$. The open circle represents the data for the PMMA-Pe chains in the bulk state.

the data for the ultra-thin film sample with thickness range of $1-100 \mathrm{~nm}$. The results clearly indicate that chain dimension in the lateral direction is not dependent on the film thickness in the ultra-thin film region. Now we compare $R_{\mathrm{xy}}$ between the ultra-thin film and the bulk state. For the measurement of the PMMA chain in an unperturbed conformation, a sample thicker than several hundred $\mathrm{nm}$ should be used because of the large molecular weight of PMMA-Pe used here. However, the SNOM imaging of a thick sample is practically difficult due to the limited penetration depth of the near-field illumination from the film surface. Therefore, we prepared the thick PMMA sample $(1 \mu \mathrm{m})$ where the PMMA-Pe chains were located near the surface in an unperturbed conformation. ${ }^{41}$ The value of $R_{\mathrm{xy}}$ in the bulk state was evaluated to be $138 \mathrm{~nm}$. The chain dimension evaluated by SNOM is larger than that measured by SANS because of the different instrument function for the estimation of the structure. The open circle in Figure 7 indicates $R_{\mathrm{xy}}$ in the three-dimensional bulk. As clearly shown in this figure, the value of $R_{\mathrm{xy}}$ in bulk is almost the same value as that in the ultra-thin film state. This result indicates that the chain dimension in the parallel directions to the film surface is not dependent on the film thickness. Although the polymer chain is spatially restricted in the height direction, the radius of gyration of the chain normal to the restriction remains the similar dimension in the unperturbed state. This result is consistent with the previous studies on the conformation of PS using SANS. ${ }^{19,20}$ The recent Monte Carlo simulation showed the stretched dimension by $\sim 40 \%$ in the parallel direction to the film surface in the quasi-two-dimensional region with the thickness less than approximately $R_{\mathrm{g}} / 3 .^{15,42}$ In the current direct observation of the single chain, it should be noted that the similar value of $R_{\mathrm{xy}}$ was obtained for the monolayer sample, where the PMMA chain was strictly restricted in a twodimensional plane. This result indicates that the PMMA chain takes a contracted conformation in the two-dimensional state.

In a three-dimensional bulk state, the polymer chain takes a random coil conformation. In terms of the conformation of an individual chain, the single PMMA chain with a molecular weight of $10^{6}$ occupies the space larger than $100 \mathrm{~nm}$. Consid- 
(a)

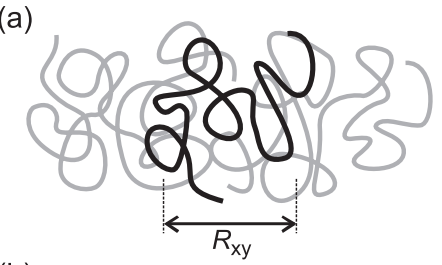

(b)

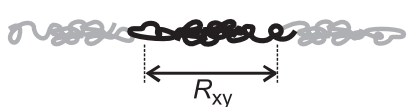

Figure 8. Schematic drawing of the chain conformations in the bulk (a) and ultra-thin film (b). To clarify the contour of the single chain, one chain is indicated as the solid curve. Few entanglements among the chains exist in the ultra-thin film, whereas there are a lot of entanglements in the bulk state.

ering the molecular volume of the single chain, there is much free space to allow the intrusion of the neighboring chains as schematically shown by the solid contour in Figure 8a. In the bulk system, such free space is filled with the surrounding chains (the gray curves in Figure 8a), resulting in entanglement of the polymer chains. Under the spatial confinement in the ultra-thin film, on the other hand, the direct observation of the single PMMA chain revealed that an in-plane dimension was similar to that in the bulk state in spite of the spatial confinement in the height direction, indicating the decrease of the pervaded volume of a single chain. Since the mass density of the thin film is almost the same as that in the bulk state ${ }^{43}$ this result indicates that the single chain does not have enough free space occupied by the neighboring chains as shown in Figure $8 \mathrm{~b}$. Consequently the entanglement among the surrounding polymer chains is reduced in the ultra-thin film. ${ }^{9,42,44}$ The direct observation of the single PMMA chain indicates little interaction between the polymer chains in the ultra-thin film. Such the characteristic conformation in the confined geometry results in unique macroscopic properties different from those of the bulk material. ${ }^{8,45}$

\section{CONCLUSION}

The real-space imaging by SNOM revealed the conformation of the single PMMA chain confined in the ultra-thin film, the thickness of which was less than the unperturbed chain dimension. In the thickness range of $1-100 \mathrm{~nm}$, the radius of gyration in the direction normal to the confinement was not significantly different from the unperturbed dimension in the three-dimensional bulk state. This indicates that the polymer chain restricted in an ultra-thin film has few entanglements with the surrounding chains.

Acknowledgment. This work was supported by Grants-inAid from Japan Society for the Promotion of Science (JSPS) and from Ministry of Education, Culture, Sports, Science and Technology (MEXT), Japan. The Innovative Techno-Hub for Integrated Medical Bio-imaging Project of the Special Coordination Funds for Promoting Science and Technology from
MEXT is also acknowledged. This work was partly supported by Tokuyama Science Foundation.

Received: November 22, 2007

Accepted: December 10, 2007

Published: January 29, 2008

\section{REFERENCES AND NOTES}

1. J. L. Keddie, R. A. L. Jones, and R. A. Cory, Faraday Discuss., 219 (1994).

2. J. L. Keddie, R. A. L. Jones, and R. A. Cory, Europhys. Lett., 27, 59 (1994).

3. T. Kajiyama, K. Tanaka, and A. Takahara, Macromolecules, 30, 280 (1997).

4. K. Tanaka, A. Taura, S. R. Ge, A. Takahara, and T. Kajiyama, Macromolecules, 29, 3040 (1996).

5. K. Tanaka, Y. Tsuchimura, K. Akabori, F. Ito, and T. Nagamura, Appl. Phys. Lett., 89, 061916 (2006).

6. O. K. C. Tsui and H. F. Zhang, Macromolecules, 34, 9139 (2001).

7. Y. Grohens, L. Hamon, G. Reiter, A. Soldera, and Y. Holl, Eur. Phys. J. E, 8, 217 (2002).

8. L. Si, M. V. Massa, K. Dalnoki-Veress, H. R. Brown, and R. A. L. Jones, Phys. Rev. Lett., 94, 127801 (2005).

9. P. G. de Gennes, "Scaling Concepts in Polymer Physics," Cornell University Press, Ithaca, New York, 1979.

10. S. K. Kumar, M. Vacatello, and D. Y. Yoon, J. Chem. Phys., 89, 5206 (1988).

11. J. Reiter, G. Zifferer, and O. F. Olaj, Macromolecules, 22, 3120 (1989).

12. I. A. Bitsanis and G. ten Brinke, J. Chem. Phys., 99, 3100 (1993).

13. A. Yethiraj, Macromolecules, 36, 5854 (2003).

14. M. Müller, J. Chem. Phys., 116, 9930 (2002).

15. A. Cavallo, M. Muller, J. P. Wittmer, A. Johner, and K. Binder, J. Phys.: Condens. Matter, 17, S1697 (2005).

16. J. Kraus, P. Muller-Buschbaum, T. Kuhlmann, D. W. Schubert, and M. Stamm, Europhys. Lett., 49, 210 (2000).

17. A. Brulet, F. Boue, A. Menelle, and J. P. Cotton, Macromolecules, 33, 997 (2000).

18. K. Shuto, Y. Oishi, T. Kajiyama, and C. C. Han, Macromolecules, 26, 6589 (1993).

19. R. L. Jones, S. K. Kumar, D. L. Ho, R. M. Briber, and T. P. Russell, Nature, 400, 146 (1999).

20. R. L. Jones, S. K. Kumar, D. L. Ho, R. M. Briber, and T. P. Russell, Macromolecules, 34, 559 (2001).

21. B. Maier and J. O. Radler, Phys. Rev. Lett., 82, 1911 (1999).

22. B. Maier and J. O. Radler, Macromolecules, 33, 7185 (2000).

23. N. Sato, Y. Osawa, S. Ito, and M. Yamamoto, Polym. J., 31, 488 (1999).

24. H. Aoki, M. Anryu, and S. Ito, Polymer, 46, 5896 (2005).

25. X. R. Wang and V. J. Foltz, J. Chem. Phys., 121, 8158 (2004).

26. W. A. Kuhlman, E. A. Olivetti, L. G. Griffith, and A. M. Mayes, Macromolecules, 39, 5122 (2006).

27. S. S. Sheiko, Adv. Polym. Sci., 151, 61 (2000).

28. J. Kumaki and T. Hashimoto, J. Am. Chem. Soc., 120, 423 (1998).

29. J. Kumaki, T. Kawauchi, and E. Yashima, Macromolecules, 39, 1209 (2006).

30. E. Betzig and J. K. Trautman, Science, 257, 189 (1992).

31. "Near-Field Nano/Atom Optics and Technology," M. Ohtsu, Ed., Springer, Tokyo, 1998.

32. M. A. Paesler and P. J. Moyer, in "Near-Field Optics: Theory, Instrumentation, and Applications," John Wiley \& Sons, New York, 1996.

33. R. C. Dunn, Chem. Rev., 99, 2891 (1999).

34. H. Aoki, Y. Sakurai, S. Ito, and T. Nakagawa, J. Phys. Chem. B, 103, 10553 (1999). 
35. H. Aoki and S. Ito, J. Phys. Chem. B, 105, 4558 (2001).

36. H. Aoki, T. Hamamatsu, and S. Ito, Appl. Phys. Lett., 84, 356 (2004).

37. S. Ito and H. Aoki, Adv. Polym. Sci., 182, 131 (2005).

38. H. Aoki, S. Tanaka, S. Ito, and M. Yamamoto, Macromolecules, 33, 9650 (2000).

39. G. Gabrielli, M. Puggelli, and P. Baglioni, J. Colloid Interface Sci., 86, 485 (1982)

40. J. M. O'Reilly, D. M. Teegarden, and G. D. Wignall, Macromolecules, 18, 2747 (1985).

41. The unlabeled PMMA was cast from the toluene solution to form a $1-\mu \mathrm{m}$ thick film on a glass substrate. A spin-cast film containing the labeled PMMA with a thickness of $70 \mathrm{~nm}$ was floated on pure water and transferred onto the thick film of the unlabeled PMMA. Then it was covered with the 40-nm spin cast film of the unlabeled PMMA The sample film was annealed at $150^{\circ} \mathrm{C}$ for a few days to reach equilibrium. From a separate experiment, the translational diffusion coefficient is estimated to be $10^{-21} \mathrm{~cm}^{2} \mathrm{~s}^{-1}$; therefore, the labeled PMMA chain is located near the surface with an equilibrium conformation.

42. H. Meyer, T. Kreer, A. Cavallo, J. P. Wittmer, and J. Baschnagel, Eur. Phys. J. Special Topics, 141, 167 (2007).

43. W. E. Wallace, N. C. B. Tan, W. L. Wu, and S. Satija, J. Chem. Phys., 108, 3798 (1998)

44. H. R. Brown and T. P. Russell, Macromolecules, 29, 798 (1996).

45. N. Sato, S. Ito, and M. Yamamoto, Macromolecules, 31, 2673 (1998). 